\title{
Impact of Disturbed Wound Healing after Surgery on the Prognosis of Marjolin's Ulcer
}

\author{
Jae Yeon Choi, Yong Chan Bae, Su Bong Nam, Seong Hwan Bae \\ Department of Plastic and Reconstructive Surgery, Pusan National University Hospital, Pusan National University School of Medicine, \\ Busan, Korea
}

Background Marjolin's ulcer is known to present a high proportion of recurrence and poor prognosis compared to other kinds of skin cancer. Based on our experience, Marjolin's ulcer patients who have received reconstructive surgery present a higher proportion of recurrence when there was disturbed wound healing after surgery. The impact of disturbed wound healing after surgery on the prognosis was examined in this study.

Methods A retrospective study was carried out on 26 patients who were diagnosed with Marjolin's ulcer and received surgery in this hospital from 1996 to 2011. Histologic grading, lymph node metastasis at diagnosis, and the wound healing process were evaluated and chisquared analysis applied in order to determine the correlation with recurrence.

Results The proportion of recurrence increases in patients with a low histologic grade or lymph node metastasis at diagnosis. The proportion of recurrence is even higher when the problem occurs during the wound healing process after surgery.

Conclusions Disturbed wound healing after surgery could be used as a sign to quickly identify the recurrence of carcinoma. Therefore, in the event a problem occurs in the wound healing process after surgery, one should keep in mind that this could be a sign of the possibility of recurrence and proceed with careful observation and active diagnosis through additional physical examinations, general X-ray tests, computed tomographys, magnetic resonance imagings, and so on, to obtain an early diagnosis of recurrence.

Keywords Carcinoma, squamous cell / Skin ulcer / Wound healing / Prognosis

\author{
Correspondence: Yong Chan Bae \\ Department of Plastic and \\ Reconstructive Surgery, Pusan \\ National University Hospital, \\ Pusan National University School of \\ Medicine, 179 Gudeok-ro, Seo-gu, \\ Busan 602-739, Korea \\ Tel: +82-51-240-7086 \\ Fax: +82-51-243-9405 \\ E-mail: baeyc2@hanmail.net
}

This work was supported by a clinical research grant from Pusan National University Hospital 2013.

No potential conflict of interest relevant to this article was reported.

\section{INTRODUCTION}

Marjolin's ulcer is an aggressive and malignant tumor that creates ulcers in external wounds, with the occurrence of chronic inflammation, scarred skin, and so on. The lesion was named after a French surgeon, Jean-Nicolas Marjolin, who first described the condition in $1828[1,2]$. Although metastasis to other parts of the body is rare compared with other malignant tumors as a result of the limited supply of blood due to surrounding scars, it progresses rapidly and presents a high mortality rate once metastasis occurs [3]. A wide excision is used for the treatment of Marjolin's ulcer and chemical anti-cancer treatment or radiation treatment accompanies it when it is necessary. However, regardless of active treatment, it is known to present a high proportion of recurrence and bad prognosis compared with other kinds of skin cancer [4]. Therefore, in regards to Marjolin's ulcer, it is important to quickly identify the signs of recurrence of carcinoma after surgical removal to improve the prognosis. Representative 
factors that have an impact on the prognosis of the patient are the histologic grade of the carcinoma before the surgery and lymph node metastasis. These representative factors are known to be negative signs in the case of a low histologic grade for carcinoma and when lymph node metastasis occurs [5]. However, little is known about the signs that may quickly alert one to recurrence in post-surgery follow-up of patients. Based on our experience, Marjolin's ulcer patients who received reconstructive surgery after wide excision and reconstruction present a higher proportion of recurrence when there has been disturbed wound healing after the surgery.

The impact of disturbed wound healing after the surgery on the prognosis was examined in this study. Moreover, the impact of the histologic grade of carcinoma before the surgery and lymph node metastasis on the prognosis of Marjolin's ulcer were also examined.

\section{METHODS}

\section{Subjects}

The subjects of this study were 26 patients who were diagnosed with Marjolin's cancer and received surgery in the authors' hospital from 1996 to 2011 . There were 15 men and 11 women. The age of the patients ranged from 31 to 74 and the average age of the subjects was $51.62 \pm 12.11$.

\section{Methods}

\section{Content of study}

A retrospective study was conducted by examining the clinical charts of 26 patients who received the surgery after being diagnosed with Marjolin's ulcer. The surgical margin was 1 to 4 $\mathrm{cm}$ and during operation, a frozen section biopsy was executed as a reference to decide the resection extent. The margin and base of the final resected main mass were confirmed to be free of cancer cells. With regard to the histologic grade of carcinoma, the degree of differentiation for squamous cell carcinoma was distinguished based on the World Health Organization's histologic grading criteria. It was classified as grade I for complete differentiation and grade III for incomplete differentiation. When lymph nodes could be felt with palpation or the enlargement of the lymph nodes could be confirmed with ultrasound or computed tomography, definite diagnosis of lymph node metastasis could be made through biopsy. A problem in the wound healing process is determined to exist when the wound is not healed within 2 weeks after the surgery and lesions, including discharge, ulcers, loss of grafts or flaps, and so on, occur in the wound. The observation was made at $37.5 \pm 42.50$ months (range, 2 to 175 months) on average. The primary cancer without evidence of distant metastasis was completely resected, and showed no residual cancer cells histologically. Cancer of the same type reoccurring at the same site was defined as a recurrence and the timing of detection was defined as the timing of the recurrence.

\section{Analysis and statistics}

For the histologic grade of carcinoma at surgery and lymph node metastasis at diagnosis as well as disturbed wound healing after the surgery, the chi-squared test was used. The test-retest reliability was analyzed using Pearson's correlation coefficient. $\mathrm{P}<0.05$ was defined as statistically significant.

\section{RESULTS}

Recurrence was presented in 6 out of 26 patients in the retrospective chart review and the average timing of recurrence after surgery was $4.66 \pm 2.07$ months (range, 2 to 8 months) for those 6 patients. Among 20 out of the total 26 patients confirmed with a histologic grade, there was one case of recurrence among 12 grade I patients, one case of recurrence among 6 grade II patients, and two cases of recurrence among 2 grade III patients. Thus it was revealed that the proportion of recurrence increased when the histologic grade was low $(\mathrm{P}<0.05)$. The carcinoma recurred for 5 out of 9 patients with lymph node metastasis at diagnosis and 1 out of 17 patients without lymph node metastasis among a total of 26 patients. Thus, it was revealed that the proportion of recurrence was higher when the patient presented with lymph node metastasis at diagnosis $(\mathrm{P}<0.05)$. Out of 26 patients, 7 patients had a problematic wound healing process. Discharge was found in 5 cases, and 4 cases had ulcers. In all 7 cases, partial or entire exfoliation of grafts or flap necrosis was found. Recurrence was exhibited in 5 out of 7 patients who had disturbed wound healing after the surgery and 1 out of 19 patients who went through normal wound healing. Thus, it was revealed that the proportion of recurrence increases when a problem occurs during the post-surgical wound healing process $(\mathrm{P}<0.05)$ (Table 1).

\section{Case 1}

A 63-year-old male patient had received several surgical reconstructions after a contact burn on the buttock when he was about 20 years old. Since October 2005, he continued to have an ulcerating lesion on the buttock and squamous cell carcinoma was discovered as a result. He was referred to the plastic surgery clinic of our hospital for reconstructive surgery. The authors conducted a wide excision of the tumor with $3 \mathrm{~cm}$ margins. A frozen section biopsy and a permanent biopsy were conducted at the margins and bases. It was confirmed that the surgical mar- 
Table 1. Histologic grading, Lymph node metastasis at diagnosis, wound healing problem and recurrence

\begin{tabular}{|lcc|}
\hline Variable & No. of patients & Recurrence \\
\hline Histologic grading & 12 & $1(8.3)$ \\
$\quad$ Grade I & 6 & $1(16.7)$ \\
$\quad$ Grade II & 2 & $2(100)$ \\
$\quad$ Grade III & & \\
Lymph node metastasis at diagnosis & 17 & $1(5.9)$ \\
$\quad$ None & 9 & $5(55.6)$ \\
$\quad$ Metastasis & & \\
Wound healing process & 19 & $1(5.3)$ \\
$\quad$ Usual & 7 & $5(71.4)$ \\
$\quad$ Unusual & & \\
\hline Values are presented as number (\%). & & \\
\hline
\end{tabular}

\section{Fig. 1. Ulcerating lesion on the buttock}

Preoperative photograph showing Marjolin's ulcer on the right side buttock.

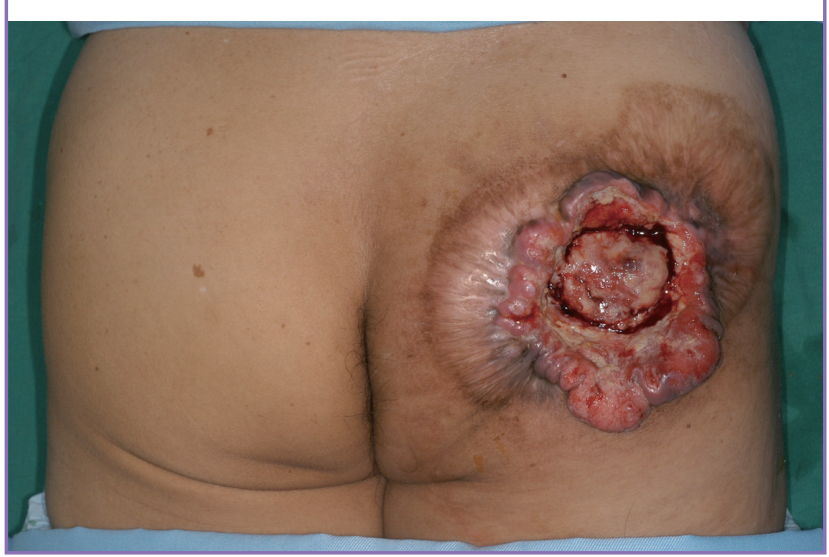

gins were free of tumor cells.

Although the skin graft was performed in November 2005, the graft failed. Surgeries (including skin graft, local flap) were conducted three additional times, and recurrence persisted. Although the patient was referred for chemical anti-cancer treatment and radiation treatment, the patient expired (Figs. 1-3).

\section{Case 2}

A 55-year-old woman suffered a flame burn injury 53 years prior to admission. She continued to have chronic ulceration, which was treated at the hospital by dressing. She presented to the plastic surgery clinic at our hospital in April 2006 with an enlarging ulceration. A biopsy was conducted and squamous cell carcinoma was identified. A wide excision and free anterolateral thigh flap were conducted for reconstruction of the heel defect in May 2006. A frozen section biopsy and permanent biopsy were conducted at the margins and bases. It was confirmed that the surgical margins were free of tumor cells.

During her follow-up, wound dehiscence occurred on the heel
Fig. 2. Disturbed wound healing lesion on the buttock

One month postoperatively, wide excision and a skin graft was performed, but there was skin graft failure.

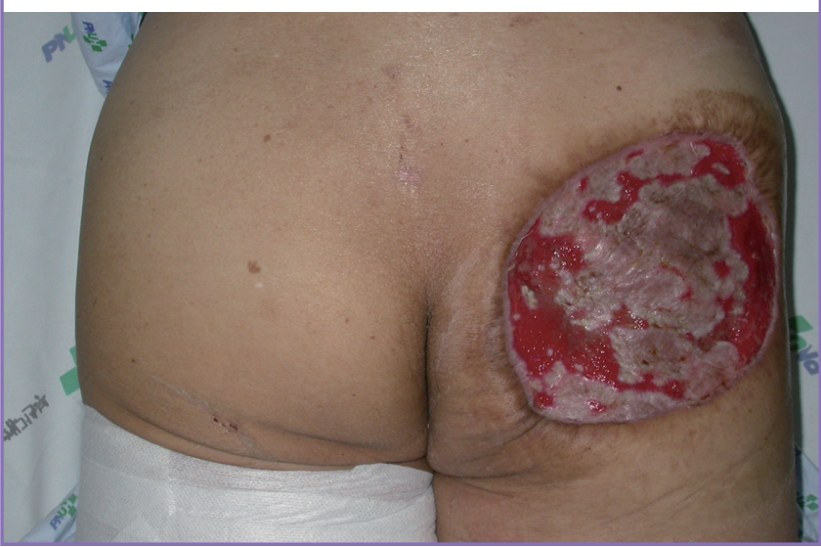

Fig. 3. Recurrence of ulcerating lesion on the buttock

Three additional operations were performed, but recurrence of carcinoma was discovered in a biopsy conducted as a result of the recurrence of the ulcerating skin lesion.

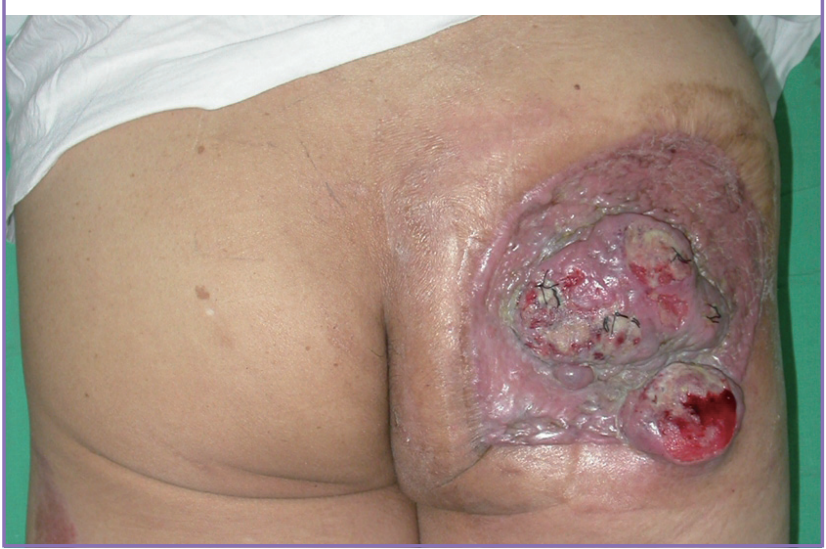

and recurrent squamous cell carcinoma was presented. A below knee amputation was conducted with chemical anti-cancer treatment and radiation treatment for therapeutic possibilities, but the patient expired (Figs. 4, 5).

\section{DISCUSSION}

When the wound healing period lengthens due to the a lack of proper treatment, severe scars left behind and chronic ulcers occur due to repeated injury, particularly in the protrusion of the bone or joint. In such ulcers, repeated incomplete epithelialization attempts, called Marjolin's ulcer, cause malignant degeneration after a long period of time [6]. Marjolin's ulcer refers to all malignant tumors that occur in chronic ulcers, scars, and inflammation areas $[2,7]$. Jean Nicholas Marjolin first illustrated tumorous changes in chronic burn scars in 1828 [2]. Treatment involves a wide surgical excision with a range of at least $2 \mathrm{~cm}$, in- 


\section{Fig. 4. Ulcerating lesion on the heel}

Preoperative photograph showing Marjolin's ulcer on the right side heel.

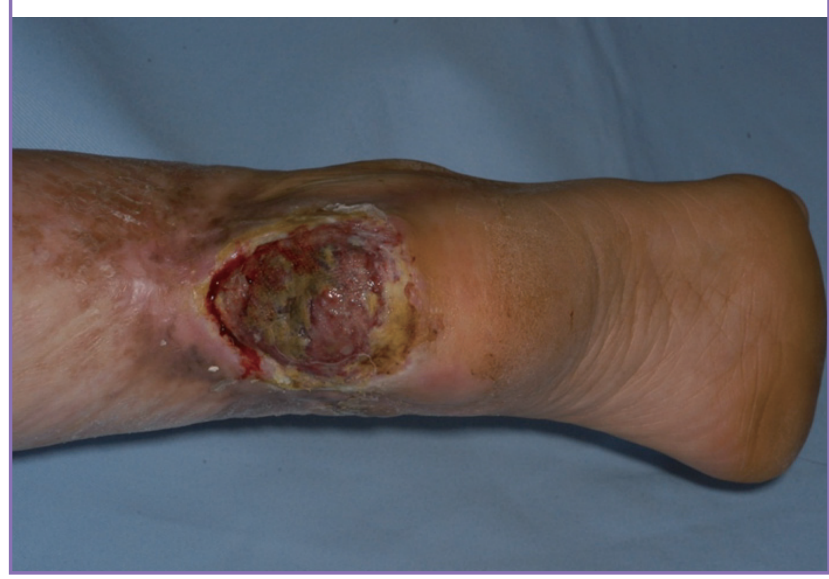

\section{Fig. 5. Disturbed wound healing lesion on the heel}

Two months postoperatively, wound dehiscence occurred and squamous cell carcinoma was discovered with tibia and lung metastasis.

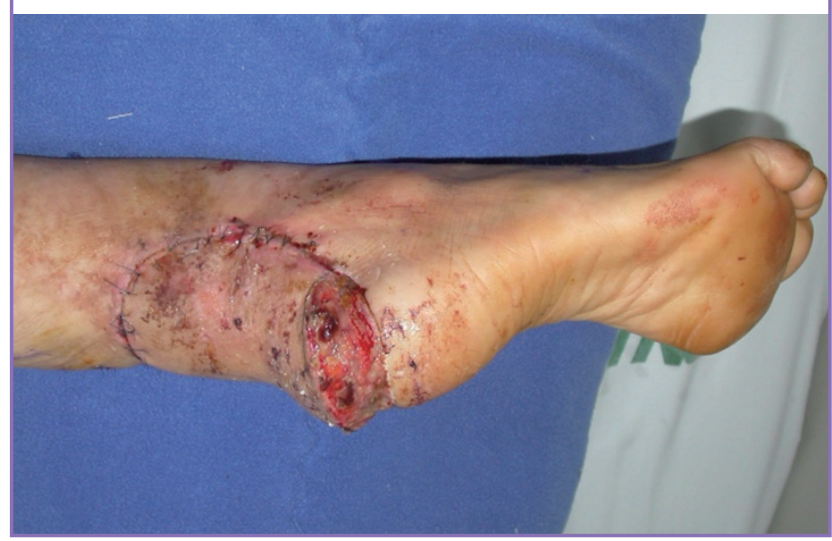

cluding thick scar tissue in the margin of the ulcer [8]. The size of the tumor is decreased with chemotherapy before the surgery and chemotherapy is conducted after the surgical excision as a combination therapy [9]. Although the cure rate is high after the operation, in cases in which there is no lymph node metastasis, the cancer progresses quickly and leads to death within 2 years once metastasis occurs [8]. Regardless of active treatment, a higher proportion of recurrence and poor prognosis are exhibited for Marjolin's ulcer compared to other skin cancers [6]. Although representative factors that have an impact on the prognosis of the patient are the histologic grade of the carcinoma before the surgery and lymph node metastasis, little is known about the signs that can quickly identify the recurrence with regard to follow-up treatment. While concentrating on the importance of the surgical resection margin, Bozkurt et al. [10] reported that the aggressive approach is able to improve prognosis based on
Table 2. Demographic data, causative previous lesions, previous therapy, latent period, site of lesions, and length of follow-up

\begin{tabular}{|llcc|}
\hline Variable & Response & $\begin{array}{c}\text { None recurred } \\
(\mathbf{n}=\mathbf{2 0})\end{array}$ & $\begin{array}{c}\text { Recurred } \\
(\mathbf{n}=\mathbf{6})\end{array}$ \\
\hline Demographic & Male & 13 & 2 \\
data & Female & 7 & 4 \\
Causative & Mean age & 52.25 & 49.5 \\
previous & Burn & 16 & 6 \\
lesions & Traffic accident & 2 & 0 \\
& Pressure sore & 1 & 0 \\
Previous therapy & No record & 1 & 0 \\
& Skin graft & 14 & 3 \\
& Conservative therapy & 6 & 2 \\
Latent period (yr) & No record & 0 & 1 \\
& 21-30 & 2 & 0 \\
& $31-40$ & 3 & 3 \\
& $41-50$ & 5 & 0 \\
& 51 & 5 & 1 \\
& No record & 4 & 2 \\
& Mean & 1 & 0 \\
& & $41 \pm 15.45$ & $37.9 \pm 13.74$ \\
Site of lesions & Head & (range, 22 to 64$)$ & (range, 13 to 64) \\
& Trunk & 3 & 2 \\
& Upper extremity & 4 & 1 \\
& Lower extremity & 7 & 1 \\
& & & 2 \\
\hline
\end{tabular}

the fact that there was only 1 recurrence out of 16 cases with a 5 $\mathrm{cm}$ surgical margin. At the same time, Chalya et al. [11] reported that early recognition, aggressive treatment, and close follow up are helpful in the improvement of the prognosis, considering the medical treatment conditions of northwestern Tanzania.

Therefore, it is important to take an aggressive approach and quickly notice the recurrence after surgery for Marjolin's ulcer to improve the prognosis of the patient. As shown in the results of this study, disturbed wound healing has a statistically significant relationship to the recurrence of carcinoma. In case a problem occurs in the wound healing process after surgery, active diagnosis and treatment may be urgently needed to improve the prognosis. With regard to the recurrence of carcinoma, Aydogdu et al. [12] reported that, on average, 8 out of 12 patients experienced recurrence within 5.4 months (range, 3 to 10 months) after the surgery, and Onah et al. [13] reported that there was recurrence in 11 out of 25 patients, including 5 cases of recurrence within 3 months, 2 cases of recurrence within 6 months, and 4 cases of recurrence within 1 year after the surgery. In our case, there was recurrence within $4.66 \pm 2.07$ months (range, 2 to 8 months). The short period of time from the removal of carcinoma to its recurrence should be taken into consideration.

A comparison of the clinical characteristics of the patients has been conducted between the recurring group and nonrecurring group. During the period under study, 13 males and 
7 females were in the non-recurring group while 2 males and 4 females were in the recurring group. Burns were the most common causative lesions in the recurring group and non-recurring group. Most primary wound sites were healed by skin graft. The extremities were the most common sites of Marjolin's ulcer (6 in the upper extremities and 7 in the lower extremities in the non-recurring group; 1 in the upper extremities and 2 in the lower extremities in the recurring group). The latent period was $41 \pm 15.45$ years (range, 22 to 64 years) in the non-recurring group and $37.9 \pm 13.74$ years (range, 13 to 64 years) in the recurring group, but statistical analysis was not possible (Table 2).

Recurrence at the same site of the surgery within a short time, strictly speaking, may mean that complete resection was not performed. However, depending on the results of the frozen section biopsy, the authors determined the extent of the surgery and the resection was performed with 1 to $4 \mathrm{~cm}$ margins. It was also confirmed that there were no cancer cells in a permanent biopsy on the resection margin and base of the final resected main mass. The authors have no doubt that complete resection was performed clinically in all possible cases. Nevertheless, given the higher proportion of recurrence, it is assumed that our clinically complete resection may not have been adequate treatment.

The results of this study do not show delayed wound healing as a prognostic factor because this study could not exclude other factors due to the lack of a sufficient number of cases. The point of this paper is that, in cases in which a problem occurs in the wound healing process after surgery, one should keep in mind that there is a possibility of recurrence of carcinoma and active treatment is urgently needed to improve the prognosis.

As a result of conducting a retrospective study on the clinical chart of 26 Marjolin's ulcer patients treated in the plastic surgery clinic of our hospital, it was revealed that the proportion of recurrence increases when the histologic grade of carcinoma is low or lymph node metastasis is presented at diagnosis, which is consistent with the previous literature. Furthermore, it was shown that disturbed wound healing after surgery has a statistically significant relationship to the recurrence of carcinoma.

A limitation of this study is the lack of a sufficient number of cases. Nonetheless, the evidence shows that in the case a problem occurs in the wound healing process after surgery, there is a higher possibility of recurrence. To assist in the treatment of the patient, careful observation and active diagnosis should be conducted through additional examinations to obtain early diagnosis of recurrence.

\section{REFERENCES}

1. Simmons MA, Edwards JM, Nigam A. Marjolin's ulcer presenting in the neck. J Laryngol Otol 2000;114:980-2.

2. Browne HR. Marjolin's ulcer: report of a case. Am J Surg 1941;54:466-71.

3. Bostwick J 3rd, Pendergrast WJ Jr, Vasconez LO. Marjolin's ulcer: an immunologically privileged tumor? Plast Reconstr Surg 1976;57:66-9.

4. Copcu E. Marjolin's ulcer: a preventable complication of burns? Plast Reconstr Surg 2009; 124:156e-64e.

5. Tiftikcioglu YO, Ozek C, Bilkay U, et al. Marjolin ulcers arising on extremities. Ann Plast Surg 2010;64:318-20.

6. Treves N, Pack GT. The development of cancer in burn scars: an analysis and report of thirty-four cases. Surg Gynecol Obstet 1930;51:749-82.

7. Kim AJ, Kuh JH, Kim KS. Malignant fibrous histiocytoma of the chest wall. Korean J Thorac Cardiovasc Surg 1996;29: 472-5.

8. Novick M, Gard DA, Hardy SB, et al. Burn scar carcinoma: a review and analysis of 46 cases. J Trauma 1977;17:809-17.

9. Sadek H, Azli N, Wendling JL, et al. Treatment of advanced squamous cell carcinoma of the skin with cisplatin, 5-fluorouracil, and bleomycin. Cancer 1990;66:1692-6.

10. Bozkurt M, Kapi E, Kuvat SV, et al. Current concepts in the management of Marjolin's ulcers: outcomes from a standardized treatment protocol in 16 cases. J Burn Care Res 2010;31:776-80.

11. Chalya PL, Mabula JB, Rambau P, et al. Marjolin's ulcers at a university teaching hospital in Northwestern Tanzania: a retrospective review of 56 cases. World J Surg Oncol 2012; $10: 38$.

12. Aydogdu E, Yildirim S, Akoz T. Is surgery an effective and adequate treatment in advanced Marjolin's ulcer? Burns 2005;31:421-31.

13. Onah II, Olaitan PB, Ogbonnaya IS, et al. Marjolin's ulcer (correction of ucler) at a Nigerian hospital (1993-2003). J Plast Reconstr Aesthet Surg 2006;59:565-6. 